\title{
Subjectivity of time perception: a visual emotional orchestration
}

\author{
Anna Lambrechts ${ }^{1}$, Nathalie Mella ${ }^{2}$, Viviane Pouthas ${ }^{3}$ and Marion Noulhiane ${ }^{4}$ * \\ ${ }^{1}$ INSERM Cognitive Neuroimaging Unit (U992), CEA/DSV/I2BM/NeuroSpin, Gif-sur-Yvette, France \\ ${ }^{2}$ Department of Psychology, University of Geneva, Geneva, Switzerland \\ ${ }^{3}$ Groupe Cogimage CRICM, UPMC/INSERM UMRS975/CNRS UMR7225, Paris, France (retired) \\ ${ }^{4}$ UMR 663 Epilepsies de I'Enfant et Plasticité Cérébrale - INSERM Paris Descartes University Hôpital Necker Enfant Malade, France /CEA/DSV/I2BM/NeuroSpin, \\ Gif-sur-Yvette, France
}

\section{Edited by:}

Valerie Doyere, Centre National de la

Recherche Scientifique, France

\section{Reviewed by:}

Annett Schirmer, National University

of Singapore, Singapore

Kwang-Hyuk Lee, University of

Sheffield, UK

${ }^{*}$ Correspondence:

Marion Noulhiane, UMR663 INSERM Université Paris Descartes -

Epilepsies de l'Enfant et Plasticité Cérébrale Hôpital Necker Enfant

Malade / CEA NeuroSpin, I2BM, DSV, CEA, Bat 145, PC 156 - 91191 Gif Sur Yvette, France.

e-mail: marion.noulhiane@ parisdescartes.fr
The aim of the present study was to examine how visual emotional content could orchestrate time perception. The experimental design allowed us to single out the share of emotion in the specific processing of content-bearing pictures, i.e., real-life scenes. Two groups of participants had to reproduce the duration $(2,4$, or $6 s)$ of content-deprived stimuli (gray squares) or differentially valenced content-bearing stimuli, which included neutral, pleasant, and unpleasant pictures (International Affective Pictures Systems). Results showed that the effect of content differed according to duration: at $2 \mathrm{~s}$, the reproduced duration was longer for content-bearing than content-deprived stimuli, but the difference between the two types of stimuli decreased as duration increased and was not significant for the longest duration (6s). At $4 \mathrm{~s}$, emotional (pleasant and unpleasant) stimuli were judged longer than neutral pictures. Furthermore, whatever the duration, the precision of the reproduction was greater for non-emotional than emotional stimuli (pleasant and unpleasant). These results suggest a dissociation within content effect on timing in the visual modality: relative overestimation of all content-bearing pictures limited to short durations ( $2 \mathrm{~s})$, and delayed overestimation of emotional relative to neutral pictures at $4 \mathrm{~s}$, as well as a lesser precision in the temporal judgment of emotional pictures whatever the duration. Our results underline the relevance for time perception models to integrate two ways of assessing timing in relationship with emotion: accuracy and precision.

Keywords: subjective time, content, emotional valence

\section{INTRODUCTION}

The literature on time perception contains many examples showing that subjective time is greatly modulated by stimulus features. A well-known effect is the sensory modality effect, i.e., the duration of light and sound are judged differently. Evidence suggests that auditory stimuli are judged longer and with greater precision than visual stimuli of equal duration and these findings have generally been interpreted in the framework of the scalar expectancy theory (Gibbon et al., 1984; Penney and Tourret, 2005; Droit-Volet et al., 2007; Noulhiane et al., 2008). This latter considers the model of an internal clock composed of three stages (1) a clock, i.e., a switch opening at the beginning of a temporal event and closing at the end of it, and a pacemaker generating pulses, (2) an accumulator stocking pulses throughout the event, (3) a comparator computing the difference between the accumulated number of pulses in working memory and a known duration in reference memory. In this interpretation the modality effect is mainly located at the clock stage: either because the pacemaker is faster in the auditory modality (Wearden et al., 1998, 2006; Droit-Volet et al., 2004b), or because the switch flickers more in the visual modality, more pulses are accumulated with sounds than lights therefore auditory signals seem longer than visual stimuli of equivalent duration (Collier and Logan, 2000; Penney et al., 2000; Droit-Volet et al., 2007). Another effect involves the sensory material filling the interval to be timed. In the auditory modality, a clear distinction is made between empty and filled intervals: empty intervals are bounded by two brief tones, whereas filled intervals contain a continuous tone with a beginning and an end. Psychophysical studies indicate greater accuracy in the timing of empty intervals (Rammsayer and Lima, 1991; Grondin, 1993). In addition, an increasing number of studies show that numerical and spatial content can interfere with temporal estimation, i.e., the number and the size of objects presented, or the magnitude of task-irrelevant Arabic numbers appearing alongside the stimulus modulate temporal judgment: the larger the number or size, the longer the subjective duration (Roitman et al., 2007; Casasanto and Boroditsky, 2008; Oliveri et al., 2008; Bueti and Walsh, 2009). A last feature of interest concerns the influence of emotion on time perception.

A recent upsurge in the number of studies on the research of time perception involves the probing of emotional stimuli on perceived duration. These studies are probably inspired by everyday knowledge of how in unpleasant situations we experience a slower passage of time and overestimate duration. This aspect of time perception, although part of everyday experience, had been neglected in research over the past few decades. Only recently, a body of evidence has been accumulated that is indicative of the intricate 
interplay between mood states and perceived duration. That is, in psychophysical experiments auditory or visual stimuli that have emotional content are often overestimated when compared to neutral stimuli. Studies using emotional visual stimuli such as facial expressions or standardized emotional pictures from International Affective Pictures Systems (IAPS, Lang et al., 1997), or standardized emotional sounds from International Affective Digital Sounds (Bradley and Lang, 2007), indicate that duration perception is highly dependent on emotional content. For instance, Angrilli et al. (1997) proposed that emotion should not be considered as a whole but as the combination of two factors: valence (i.e., the sign of the emotional value) and intensity (i.e., the magnitude of the emotional value). They designed a paradigm to disentangle the effect of each component on time perception. Participants were presented with pleasant and unpleasant pictures, both moderately and highly arousing, alongside neutral pictures from the IAPS. Pictures appeared for 2, 4, or $6 \mathrm{~s}$. Participants were asked to perform a prospective timing task, either by indicating the perceived duration of the picture presentation on an analog scale or by pressing a button to reproduce the duration. The analysis showed an interaction between the two components of emotion regardless of the timing method: duration of highly arousing pleasant pictures was more underestimated than that of highly arousing unpleasant pictures whereas duration of low-arousing pleasant pictures was less underestimated than that of low-arousing unpleasant pictures. However, no clear comparison with neutral stimuli, which were used as fillers, was performed. Yet durations seem subjectively different when timing stimuli with an emotional valence than when timing stimuli with a neutral valence (e.g., Watts and Sharrock, 1984; Droit-Volet et al., 2004a; Gil et al., 2007; Noulhiane et al., 2007). Overall, duration of all emotional pictures is overestimated in comparison with that of neutral pictures. In the framework of an internal pacemaker model researchers postulated that such emotional overestimation was mainly due to an arousal-related acceleration of the internal pace in such situations. Thus overestimation is greater for highly arousing emotions (for instance, angry faces are the most overestimated, followed by happy and then sad faces; Droit-Volet and Gil, 2009). Attention is also believed to impact timing: when attention is diverted from the timing task, i.e., by a concurrent task or by emotional information, it can delay the switch closure or produce a flickering which both lead to a fewer number of accumulated pulses, and therefore an underestimation of duration (Droit-Volet and Gil, 2009; Lui et al., 2011). Although there is increasing interest in the subjective experience of time and how it is modulated by emotional states, to our knowledge, no study has compared stimuli with a content (i.e., ecological stimuli, capturing some of the complexity of reallife scenes) and those without (e.g., a picture of a square or a pure tone). The existence of such a valence rating as neutral is somewhat controversial since it is not clear whether there is such an emotional category or whether neutral stimuli simply have a lowvalue valence or emotional intensity. The use of content-deprived stimuli would provide a higher contrast between emotional and non-emotional pictures - and thus would test the heuristic value of timing model to determine the influence of emotion. Finally, the impact of emotions on subjective time has been largely investigated in short-time scale subjective duration (i.e., sub-second to second range), with most findings assuming an internal pacemaker devoted to time. Studies using longer scales are still scarce and do not systematically reinforce the internal pacemaker theory. Alternative concepts favor the idea of duration-dependant timing processes (Vierordt, 1868; Fraisse, 1984; Pöppel, 2009; Wittmann and van Wassenhove, 2009).

The aim of the present study was to investigate how visual emotional content influences time perception. By content we will refer hereafter to the property for a picture to be realistic, i.e., to contain representation of real-life objects or scenes, as opposed to abstract signs or shapes. We used a temporal reproduction paradigm involving stimuli of gradual content-related emotional valence (content-deprived, content-bearing neutral, pleasant, and unpleasant pictures). Two groups of participants were presented with three standard durations (2, 4, and $6 \mathrm{~s}$ ) of visual stimuli: (1) Group 1 had to encode the duration of a content-deprived stimulus, i.e., a gray square (no-content task); (2) Group 2 had to encode the duration of content-bearing stimuli from the IAPS, i.e., pictures rated for neutral, pleasant, and unpleasant emotional valence (content task). We assumed that the duration of content-deprived stimuli would be judged shorter than that of content-bearing stimuli. We also expected that neutral stimuli would be judged shorter than emotional ones (positive or negative valence).

\section{MATERIALS AND METHODS PARTICIPANTS}

Thirty-four right-handed participants with no history of neurological or psychiatric disorder volunteered for this study. Seventeen performed the no-content task (Group 1) and seventeen performed the content task (Group 2). All participants were recruited in a population of young adults (age range 20 to 35 years old) with a higher-education profile (Table 1). Two independent groups assumed no interference between tasks. In accordance with the Declaration of Helsinki, all participants gave their informed consent.

\section{MATERIALS}

The experiment was conducted using an IBM compatible computer with a module controlling a shutter for the presentation of visual stimuli. A specifically designed program controlled both the presentation of the standard durations and the recording of the participants' responses (reproduced durations) with an accuracy of $\pm 0.5 \mathrm{~ms}$. Stimuli were displayed in the center of the screen against a black background, at a viewing distance of $0.5 \mathrm{~m}$.

The two tasks were adapted from previous studies using either emotional content-bearing stimuli in the visual modality (Angrilli et al., 1997), content-deprived stimuli in the visual modality or emotional stimuli in the auditory modality (Noulhiane et al., 2007, 2008). For the no-content task, the stimulus was a gray

Table 1 | Demographic data of participants.

\begin{tabular}{|c|c|c|c|}
\hline & Total number & $\begin{array}{l}\text { Age } \\
\text { (mean } \pm S D, \text { years) }\end{array}$ & $\begin{array}{l}\text { Educational level } \\
\text { (mean } \pm S D, \text { years) }\end{array}$ \\
\hline Group 1 & 17 (female, 8) & $30.3 \pm 5$ & $15.27 \pm 2.3$ \\
\hline Group 2 & 17 (female, 8) & $25.2 \pm 3$ & $16.94 \pm 0.97$ \\
\hline
\end{tabular}


$10 \times 10 \mathrm{~cm}$ square of uniform luminance. For the content task, 36 pictures were selected from the IAPS (neutral: 1390, 5120, 5531, 5731, 7002, 7010, 7224, 7234, 7500, 7595, 7640, 7830; pleasant: 1340, 1463, 1500, 2170, 4659, 4670, 5480, 5629, 5831, 8080, 8180, 8190; unpleasant: 1052, 1111, 1300, 3550, 6260, 7361, 9250, $9320,9520,9584,9592,9921)$, with three 12-item groups of different valence: pleasant (mean $\pm \mathrm{SD}=7.57 \pm 0.43$ ), unpleasant $(2.84 \pm 1.13)$, and neutral $(5.57 \pm 0.66)$. In both tasks, the duration reproduction paradigm involved three standard durations: 2,4 , and $6 \mathrm{~s}$. During the no-content task each duration was presented 10 times in random order. During the content task, three series were created by selecting four pictures from each group (pleasant, unpleasant, and neutral), each picture appearing once for each duration $(2,4$, or $6 \mathrm{~s})$, adding up to 36 (picture, duration) stimuli in each list. Every participant was presented with the three lists in pseudo-random order (108 trials in all).

\section{PROCEDURE AND DESIGN}

Each trial started with a stimulus presented for a randomly selected standard duration. Participants were instructed to pay attention to the duration of the stimulus in order to reproduce it. They were told to avoid chronometric counting strategies. When the stimulus disappeared, a gray square was displayed on the screen in both tasks. Participants were asked to press the spacebar until they judged that the time elapsed equaled the duration of the picture presentation. The reproduced duration was recorded. A random 2-3s time gap was introduced after presentation of the stimulus and during the inter-trial interval to prevent participants from having an implicit evaluation of the fixed time period. Before the experiment, the participants were trained with five trials using content-deprived or neutral pictures in the no-content and content tasks respectively.

A control experiment was performed to verify that the emotions elicited by the pictures were stable over time for all durations used. Stimuli were presented for 2, 4, and $6 \mathrm{~s}$ in random order. Ratings of valence levels were collected from 12 young adults (six women, aged $27 \pm 3.43$ years, and six men, aged $27 \pm 3.77$ years) who did not participate in the main experiment. Participants were asked to rate the self-assessment Manikin. Rated scores computed for the three durations showed that subjective valence ratings matched those of the IAPS, whatever the duration. Moreover, one-way analyses of variance (ANOVA) performed on rated scores for each valence with duration as a factor revealed no significant effect of duration (all $p s>0.1$ ). In sum, emotional valence does not vary within this range of duration (Table 2).

\section{STATISTICAL ANALYSES}

Accuracy of temporal performance was examined by computing a $\mathrm{T}$-corrected score with the following formula: [ $\mathrm{T}$-corrected $=(\mathrm{T}$ estimated - $\mathrm{T}$ standard)/T standard]. Negative values indicate that the reproduced duration was shorter than the standard, and positive values indicate that it was longer. Precision of temporal performance was examined by computing the coefficient of variation $(\mathrm{CV})$ with the following formula: $[\mathrm{CV}=\mathrm{SDs}$ of mean reproduction time/mean reproduction time]. Paired $t$-tests were performed to compare results across duration (3) and content (4). Bonferroni corrections were applied for all analyses $(p<0.005)$.
Some results did not reach significance after correction $(p<0.05)$, but they are still presented hereafter for discussion. These results are however to be taken with caution.

\section{RESULTS}

\section{T-CORRECTED}

Student paired $t$-test scores (Figure 1) comparing durations within the same content showed no difference in the no-content task between 2, 4, and $6 \mathrm{~s}$. Within the content-bearing stimuli on the opposite, duration had a larger influence: all pairs of durations for each content condition were found significantly different (all ps $<0.005$ ), showing that $4 \mathrm{~s}$-pictures were underestimated with respect to $2 \mathrm{~s}$-pictures, and $6 \mathrm{~s}$-pictures were underestimated with respect to both 2 - and $4 \mathrm{~s}$-pictures for all content conditions. Overall, the longer the duration, the more underestimated it is. Note that "underestimation" is used relatively between durations.

Table 2 | Rated scores from IAPS and those from 12 participants computed for the three durations.

\begin{tabular}{llll}
\hline Duration/content & IAPS score & $\begin{array}{l}\text { Rated score } \\
( \pm \mathbf{S D})\end{array}$ & $\begin{array}{l}\text { Student test, } \\
\boldsymbol{t}(\mathbf{1 1}) ; \boldsymbol{p}\end{array}$ \\
\hline 2 s Pleasant & 7.58 & $6.22 \pm 0.62$ & $2.02 ; \mathrm{NS}$ \\
2 s Unpleasant & 2.84 & $2.68 \pm 0.38$ & $0.40 ; \mathrm{NS}$ \\
2 s Neutral & 5.57 & $4.73 \pm 0.66$ & $1.22 ; \mathrm{NS}$ \\
4 s Pleasant & 7.58 & $6.29 \pm 0.54$ & $2.22 ; \mathrm{NS}$ \\
4 s Unpleasant & 2.84 & $2.49 \pm 0.43$ & $0.75 ; \mathrm{NS}$ \\
4 s Neutral & 5,57 & $4.62 \pm 0.77$ & $1.17 ; \mathrm{NS}$ \\
6 s Pleasant & 7.58 & $6.31 \pm 0.60$ & $1.94 ; \mathrm{NS}$ \\
6 s Unpleasant & 2.84 & $2.49 \pm 0.39$ & $0.84 ; \mathrm{NS}$ \\
6 s Neutral & 5.57 & $4.72 \pm 0.81$ & $1.01 ; \mathrm{NS}$ \\
\end{tabular}

Student unpaired t-tests showed that scores were matched to IAPS scores whatever the duration. One-way ANOVAS performed for each valence with duration as a factor showed no main effect of duration [pleasant: $F(2,22)=2.22, p=0.13$; unpleasant: $F(2,22)=0.94, p=0.40$; neutral: $F(2,22)=0.65, p=0.53$ ]: emotional valence does not vary with duration. Rated scores for emotional valence.

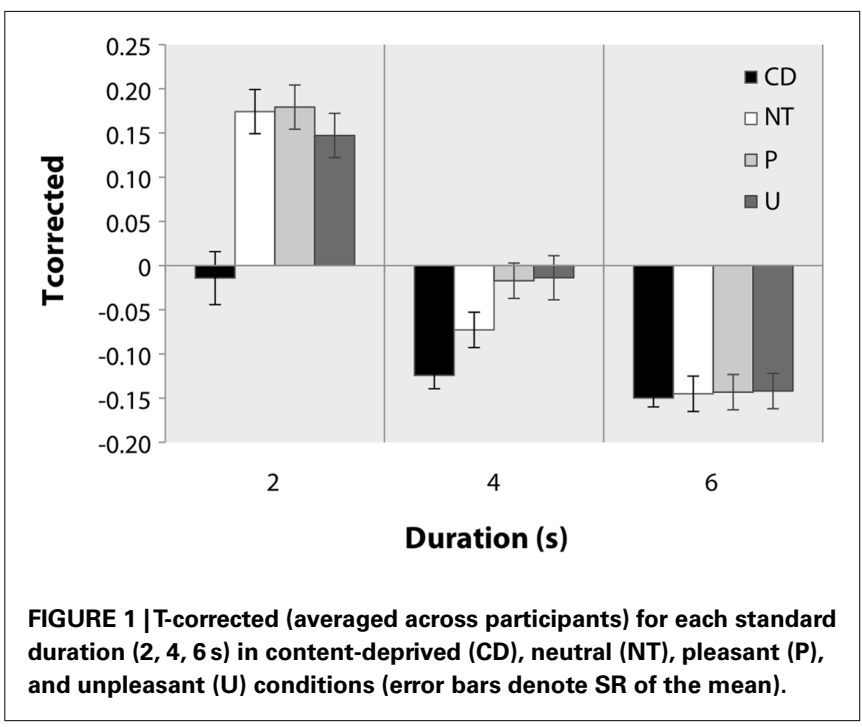


Comparison of subjective durations to their actual standards showed that $2 \mathrm{~s}$-stimuli are overestimated, $4 \mathrm{~s}$-stimuli are quite accurately reproduced, and $6 \mathrm{~s}$-stimuli are underestimated.

Student paired $t$-test scores between content conditions showed that at $2 \mathrm{~s}$, content-deprived stimuli were judged shorter than neutral and pleasant content-bearing pictures $[t(16)=-2.24$, $p=0.039$ and $t(16)=-2.18, p=0.044$, respectively]. There was no difference between any of the content conditions at $2 \mathrm{~s}$. At $4 \mathrm{~s}$, there was no difference between content-deprived and any of the content-bearing conditions. The neutral pictures were judged shorter than the pleasant and the unpleasant ones $[t(16)=-2.27$, $p=0.037$ and $t(16)=-2.12, p=0.050$, respectively]. No difference was found between pleasant and unpleasant pictures. Finally, at $6 \mathrm{~s}$, no difference between content-deprived and contentbearing stimuli was found, nor between any of the content-bearing conditions.

\section{COEFFICIENT OF VARIATION}

Student paired $t$-tests (Figure 2) showed that CVs were smaller at $6 \mathrm{~s}$ than at $2 \mathrm{~s}[t(16)=4.85, p<0.001]$ and at $4 \mathrm{~s}$ than at $2 \mathrm{~s}$ for unpleasant content only $[t(16)=4.38, p<0.001]$. Over all contents the variability in response is however little influenced by duration. Within duration, Student independent $t$-tests showed that both content-deprived and content-bearing neutral pictures had smaller CVs than pleasant and unpleasant pictures for all durations (all $p s<0.001$ ). No difference was found between content-deprived and neutral content-bearing pictures. These results showed that emotional pictures are judged with greater variability than both content-deprived and neutral pictures, even though the effect slowly diminishes with duration.

\section{DISCUSSION}

This study aimed at investigating the influence of visual emotional content on time perception. Participants reproduced 2, 4 , and $6 \mathrm{~s}$ durations in a task contrasting stimuli of varying emotional valence (content-bearing neutral, pleasant, and unpleasant pictures) with content-deprived stimuli (gray squares).

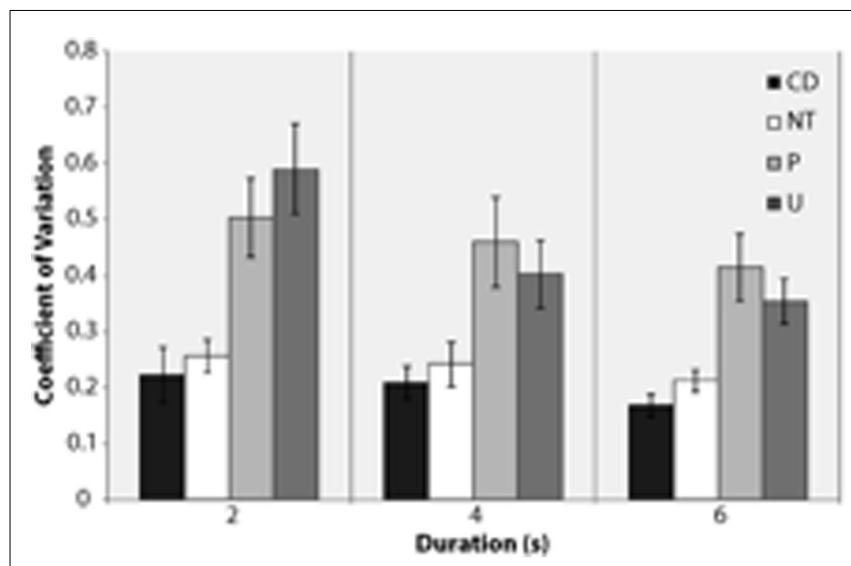

FIGURE 2 | Coefficient of variation (averaged across participants) for each standard duration $(2,4,6 \mathrm{~s})$ in content-deprived (CD) and content-bearing [neutral (NT), pleasant (P), unpleasant (U)] conditions. (Note error bars denote SR of the mean.)
The main results revealed that content affected both the accuracy and precision of the responses, and that this effect generally diminished with time.

The measures of response accuracy (T-corrected) showed that for a $2 \mathrm{~s}$ duration, all content-bearing stimuli were judged to last longer than content-deprived ones; at 4 and $6 \mathrm{~s}$, no more significant differences were found. In the framework of the internal clock model, this content-related effect can be explained by an attentional mechanism, a gray square capturing presumably less attention than a content-bearing picture (Zakay, 2005). According to this theory, enhanced attention paid to content-bearing pictures would both fasten internal pacing and reduce the switch flickering, making duration appear longer (Tse et al., 2004; Zakay, 2005; New and Scholl, 2009). This hypothesis is drawn in parallel to the idea that emotional stimuli capture attentional resources more easily than non-emotional ones (Droit-Volet and Meck, 2007). Our results revealed thus that not only emotional but more generally content-bearing stimuli (even neutrally valenced) are still more susceptible to capture attention than content-deprived stimuli. Using a gray square, we did not investigate the specific input of arousal. In future studies, this aspect would however need to be examined by controlling the arousal level induced by the presentation of content-deprived stimuli, for instance by mean of physiological measures (e.g., skin conductance response, heart rate). Additionally it should be noted that we chose to increase the contrast between content-deprived and content-bearing stimuli by using a simple one-color shape on the one hand, and complex multicolored scenic pictures on the other hand. These features are possible confounds that should be controlled for in future studies by using for instance scrambled versions of the content-bearing pictures as content-deprived stimuli. However in this context, the relevance of the IAPS was to provide a battery of pictures rated for emotion on a 1-9 linear scale (Lickert scale, $1=$ highly unpleasant, $9=$ highly pleasant). "Neutral" pictures could thus still elicit emotion in some participants (as reflected by interindividual variability, see Lang et al., 1997), whereas by using geometrical squares we made sure that the stimuli elicited no emotion at all. Our experimental design separated the content conditions into two tasks executed by two different groups. Although this methodological point could be considered as a limit, this allowed us to ensure the gray square during the reproduction phase did not trigger any change in the pacemaker rate in the content-deprived task as it could have if it had been contextualized by a majority of content-bearing pictures, had the task been intermixed. Nevertheless, futures studies should control this aspect by having the same participants perform the two tasks in a counter-balanced order, or by intermixing content-deprived and content-bearing stimuli with an equal number of trials in each condition.

Overall, we found that for any content-bearing stimulus, the longer the duration, the shorter it is judged relative to the standard. This effect of duration is consistent with Vierordt's (1868) law; in a series of durations, the shortest are over-reproduced and the longest are under-reproduced, reproduction of sample durations below and above the mean of the series shifting progressively toward the central value. Yet an interesting result is that the effect of stimulus content on time processing decreases as the duration to be processed increases. We propose that the attentional effect drawn 
by content-bearing stimuli is limited in time: attention reflects the ability to quickly allocate neural resources to the processing of a relevant stimulation. Once the latter has been processed and the appropriate answer has been produced, attentional level decreases, leading the internal pace to return to its initial speed. This lowering of content-induced activation with time could appear at odds with the literature on activation and timing. Studies have generally reported a greater activation effect for longer than for shorter times, the effect being multiplicative (Burle and Casini, 2001). As in our previous study (Noulhiane et al., 2007), the present results show the reverse pattern. However, most studies have tested the influence of activation on the processing of durations shorter than $2 \mathrm{~s}$, which was the lowest limit of our range. Moreover in the emotional context, these studies have usually used facial emotional expressions which constitute particular and unique stimuli for humans as they involved social interactions and categorical emotions (happiness, sadness, fear...). One may then posit that the pacemaker rate increases within the duration of the content effect, driven either by attention, arousal or complexity processing, and then gradually returns to its baseline. Future studies in the subsecond range using emotional scenes of real-life could clarify this point.

Focusing on the emotional valence, our results surprisingly show that emotion has a delayed effect on time reproduction: at $2 \mathrm{~s}$ pleasant and unpleasant pictures were not judged differently from the neutral pictures; however at $4 \mathrm{~s}$ they were judged longer than neutral pictures. At $6 \mathrm{~s}$, no difference was observed anymore. This result suggests that the difference in pace between non-emotional and emotional scenes is not so big that we can notice the difference after a $2 \mathrm{~s}$-accumulation, but in $4 \mathrm{~s}$ the difference is bigger and reaches significance. As mentioned above, the pace then progressively returns to its baseline level, so that at $6 \mathrm{~s}$ no emotional-driven difference is noticeable again. This result differed from those reported by Lui et al. (2011), where the authors introduced either an emotional or neutral picture from the IAPS in-between the reference (S1) and the probe (S2) for durations ranging from 1100 to $2300 \mathrm{~ms}$ in a reproduction task. They found that the duration of S2 was underestimated after an emotional stimulus compared to a neutral stimulus, in agreement to an acceleration of the pacemaker triggered by emotion. However the presentation of the task-irrelevant picture happened $1300 \mathrm{~ms}$ before the presentation of S2, overruling any latency in the emotional effect on the pacemaker, which could explain the presence of an emotional effect below $2 \mathrm{~s}$. It is also in contradiction with what was observed in the auditory modality within the same duration range as in the present study, where the effect of emotion happened early and decreased with time (Noulhiane et al., 2007). Such discrepancy raises a broader issue about the relationship between timing, emotion and sensory modality: namely, this questions whether the intrinsically dynamic aspect of auditory stimuli facilitates the extraction of emotional information in comparison to static visual stimuli, and whether this would result in a delay of emotional effect between auditory and visual modalities. Future studies using emotional cross-modality paradigm could refine this question.

The effect of stimuli content appeared also on the precision: the CVs were slightly reduced with the length of duration for any content. This effect could not be explained by the use of chronometric counting since the high values reflect timing processing (all CVs > 0.15; Wearden, 1991). Focusing on emotional valence, the variability of judgments (CVs) was greater for emotional (both pleasant and unpleasant) than non-emotional (both neutral and content-deprived) pictures, for all three standard durations. This suggests that emotional valence, and not content, reduces the precision of temporal judgments. To respond appropriately to a picture, it is more important to decipher emotional valence, and probably the nature of the conveyed emotion, than to evaluate the exact duration of its presentation. In line with the internal clock model, we hypothesize that emotion induces a greater flickering of the switch. This flickering adds a random factor that increases the variability of the final estimation. Note that this does not contradict the fact that more pulses are accumulated since, according to our attentional hypothesis, the pacemaker is still running faster than for content-deprived stimuli. The findings obtained on precision finally support the recent proposal that timing is a self-referential processing highly dependent of our emotional state (Craig, 2009): the reduced precision in the estimation of emotional stimuli duration may lie in the extreme variability one may observe in the experience of emotion. According to Craig's (2009) model, our sense of time is based on the succession of global emotional moments that are intrinsically linked to the interoceptive information integrated in the insular cortex. Considering that emotional stimuli are probably not equivalent in the generation of emotion within an individual - even in the same hedonic category - the internal state resulting in the exposition of such stimuli may vary across trials. As a result time estimates of such stimuli would also vary.

Finally, our data extend future perspective to elucidate mechanisms of time processing in link with content. For instance, the temporal overestimation of content-bearing stimuli could reflect a usual timing effect which has yet to be elucidated in current neural timing models: the difference in number and complexity between content-deprived and content-bearing pictures affects the temporal judgement (Ornstein, 1969; Schiffman and Bobko, 1974; Fraisse, 1984). Recent models propose that the passage of time can be encoded in the evolving patterns of activity in neural networks (Buonomano and Mauk, 1994; Buonomano and Merzenich, 1995; Mauk and Buonomano, 2004; Buhusi and Meck, 2005; Karmarkar and Buonomano, 2007; Ivry and Schlerf, 2008). This kind of model is all the more exciting that so far the clock model has found little support in physiology whereas this new approach is based on neural dynamics. These models suggest that the duration assigned to a stimulus reflects the change in distribution of neural activity following the stimulus presentation. To date, this hypothesis was essentially investigated with events of short-time scale subjective durations (e.g., in the time scale of tens to hundreds of milliseconds, Eagleman and Pariyadath, 2009). There is not enough evidence to indicate that similar mechanisms come into play in duration judgments at longer time scales while certain principles observed at brief time scales appear to hold at longer ones. Furthermore, these models have not to date investigated emotional stimuli. Future studies should determine their validity in wider ranges of duration and for the specific case of emotion.

In conclusion, this study shows that content modulates time perception in the visual modality. Interestingly, our results suggest 
a dissociation in the content effect on timing: relative overestimation of all content-bearing pictures for short durations ( $2 \mathrm{~s})$ and delayed effect of emotion at $4 \mathrm{~s}$, alongside less precision in the temporal judgment of emotional pictures, whatever their duration. This double susceptibility of timing toward emotion should be taken into consideration in future studies.

\section{REFERENCES}

Angrilli, A., Cherubini, P., Pavese, A., and Mantredini, S. (1997). The influence of affective factors on time perception. Percept. Psychophys. 59, 972-982.

Bradley, M. M., and Lang, P. J. (2007). The International Affective Digitized Sounds Affective Ratings of Sounds and Instruction Manual. Emotion 18. University of Florida, Gainesville, FL.

Bueti, D., and Walsh, V. (2009). The parietal cortex and the representation of time, space, number and other magnitudes. Philos. Trans. R. Soc. Lond. B Biol. Sci. 364, 1831-1840.

Buhusi, C. V., and Meck, W. H. (2005). What makes us tick? Functional and neural mechanisms of interval timing. Neuroscience 6, 755-765.

Buonomano, D. V., and Mauk, M. D. (1994). Neural Network model of the cerebellum: temporal discrimination and the timing of motor responses. Neural Comput. 6 , 38-55.

Buonomano, D. V., and Merzenich, M. (1995). A neural network model of temporal code generation and position-invariant pattern recognition. Neural Comput. 11, 103-116.

Burle, B., and Casini, L. (2001). Dissociation between activation and attention effects in time estimation: implications for internal clock models. J. Exp. Psychol. Hum. Percept. Perform. 27, 195-205.

Casasanto, D., and Boroditsky, L. (2008). Time in the mind: using space to think about time. Cognition 106, 579-593.

Collier, G. L., and Logan, G. (2000). Modality differences in short-term memory for rhythms. Mem. Cognit. $28,529-538$.

Craig, A. D. (2009). Emotional moments across time: a possible neural basis for time perception in the anterior insula. Philos. Trans. R. Soc. Lond. B Biol. Sci. 364, 1933-1942.

Droit-Volet, S., Brunot, S., and Niedenthal, P. M. (2004a). Perception of the duration of emotional events. Cogn. Emot. 18, 849-858.

Droit-Volet, S., Tourret, S., and Wearden, J. H. (2004b). Perception of the duration of auditory and visual stimuli in children and adults. Q. J. Exp. Psychol. 57A, 797-818.

Droit-Volet, S., and Gil, S. (2009). The time-emotion paradox. Philos. Trans. R. Soc. Lond. B Biol. Sci. 364, 1943-1953.

Droit-Volet, S., and Meck, W. H. (2007). How emotions colour our perception of time. Trends Cogn. Sci. 11, 504-513.

Droit-Volet, S., Meck, W. H., and Penney, T. B. (2007). Sensory modality and time perception in children and adults. Behav. Processes 74, 244-250.

Eagleman, D. M., and Pariyadath, V. (2009). Is subjective duration a signature of coding efficiency? Philos. Trans. R. Soc. Lond. B Biol. Sci. 364, 1841-1851.

Fraisse, P. (1984). Perception and estimation of time. Annu. Rev. Psychol. $35,1-36$.

Gibbon, J., Church, R. M., and Meck, W H. (1984). Scalar timing in memory. Ann. N. Y. Acad. Sci. 423, 52-77.

Gil, S., Niedenthal, P., and Droit-Volet, S. (2007). Anger and time perception in children. Emotion 7, 219-225. crimination of empty and filled intervals marked by auditory and visual signals. Percept. Psychophys. 54, 383-394. icated and intrinsic models of time perception. Trends Cogn. Sci. (Regul. Ed.) 12, 273-280.

Karmarkar, U. R., and Buonomano, D. V. (2007). Timing in the absence of clocks: encoding time in neural network states. Neuron 53, 427-438.

Lang, P. J., Bradley, M. M., and Cuthbert, B. N. (1997). "Motivated attention: affect, activation, and action," in Attention and Orienting: Sensory and Motivational Processes, eds P. J. Lang, R. F. Simons, and M. T. Balaban (Hillsdale, NJ: Erlbaum), 97-135.

Lui, M. A., Penney, T. B., and Schirmer, A. (2011). Emotion effects on timing: attention versus pacemaker accounts. PLoS ONE 6, e21829. doi:10.1371/journal.pone.0021829

Mauk, M. D., and Buonomano, D. V. (2004). The neural basis of temporal
Grondin, S. (1993). Duration dis-

Ivry, R. B., and Schlerf, J. E. (2008). Ded-

\section{ACKNOWLEDGMENTS}

We would like to address a special thank to Viviane Pouthas for her input and expertise, and for bringing us together on this project. More generally we thank her for her life-long contribution to the scientific development of her students which she did with kindness and constant support.

processing. Annu. Rev. Neurosci. 27, 307-340.

New, J. J., and Scholl, B. J. (2009). Subjective time dilation: spatially local, object-based, or a global visual experience? J. Vis. 9, 1-11.

Noulhiane, M., Mella, N., Samson, S, Ragot, R., and Pouthas, V. (2007). How emotional auditory stimuli modulate time perception. Emotion 7, 697-704.

Noulhiane, M., Pouthas, V., and Samson, S. (2008). Is time reproduction sensitive to sensory modalities? Eur. J. Cogn. Psychol. 21, 18-34.

Oliveri, M., Vicario, C. M., Salerno, S., Koch, G., Turriziani, P., Mangano, R., Chillemi, G., and Caltagirone, C. (2008). Perceiving numbers alters time perception. Neurosci. Lett. 438 , 308-311.

Ornstein, R. E. (1969). On the experience of time. Hammandsworth, UK: Penguin.

Penney, T. B., Gibbon, J., and Meck, W. H. (2000). Differential effects of auditory and visual signals on clock speed and temporal memory. Perception 26, 1770-1787.

Penney, T. B., and Tourret, S. (2005). Les effets de la modalité sensorielle sur la perception du temps Modality effects in short interval timing. Psychol. Fr. 50, 131-143.

Pöppel, E. (2009). Pre-semantically defined temporal windows for cognitive processing. Philos. Trans. R. Soc. Lond. B Biol. Sci. 364 1887-1896.

Rammsayer, T. H., and Lima, S. D. (1991). Duration discrimination of filled and empty auditory intervals: cognitive and perceptual factors. Percept. Psychophys. 50, 565-574.

Roitman, J. D., Brannon, E. M. Andrews, J. R., and Platt, M. L. (2007). Nonverbal representation of time and number in adults. Acto Psychol. (Amst) 124, 296-318.

Schiffman, H. R., and Bobko, D. J. (1974). Effects of stimulus complexity on the perception of brief temporal intervals. J. Exp. Psychol. 103, 156-159.

Tse, P. U., Intriligator, J., and Rivest, J. (2004). Attention and the subjective expansion of time. Perception 66, 1171-1189.
Vierordt, K. (1868). Der Zeitsinn nach Versuchen. Tübingen, Germany: Laupp.

Watts, F. N., and Sharrock, R. (1984). Questionnaire dimensions of spider phobia. Behav. Res. Ther. 22, 575-580.

Wearden, J. (1991). Do humans possess an internal clock with scalar timing properties? Learn. Motiv. 22, 59-83.

Wearden, J. H., Edwards, H., Fakhri, M., and Percival, A. (1998). Why "sounds are judged longer than lights": application of a model of the internal clock in humans. Psychology 51B, 97-120.

Wearden, J. H., Todd, N. P. M., and Jones, L. A. (2006). When do auditory/visual differences in duration judgements occur? Exp. Psychol. 59, 1709-1724.

Wittmann, M., and van Wassenhove, V. (2009). The experience of time: neural mechanisms and the interplay of emotion, cognition and embodiment. Philos. Trans. R. Soc. Lond. B Biol. Sci. 364, 1809-1813.

Zakay, D. (2005). Attention et jugement temporel Attention and duration judgment. Psychol. Fr. 50, 65-79.

Conflict of Interest Statement: The authors declare that the research was conducted in the absence of any commercial or financial relationships that could be construed as a potential conflict of interest.

Received: 15 June 2011; accepted: 12 October 2011; published online: 16 November 2011.

Citation: Lambrechts A, Mella N, Pouthas V and Noulhiane $M$ (2011) Subjectivity of time perception: a visual emotional orchestration. Front. Integr. Neurosci. 5:73. doi: 10.3389/fnint.2011.00073

Copyright (c) 2011 Lambrechts, Mella, Pouthas and Noulhiane. This is an openaccess article subject to a non-exclusive license between the authors and Frontiers Media SA, which permits use, distribution and reproduction in other forums, provided the original authors and source are credited and other Frontiers conditions are complied with. 